\title{
Synthesis and characterisation of chitosan nanoparticle as a potential delivery carrier
}

\author{
Noor Hidayah Ibrahima, Mas Jaffri Masarudin ${ }^{\mathrm{a}, \mathrm{d}}$, Abdul Rahman Omar ${ }^{\mathrm{b}, \mathrm{c}}$, Mohd Hair Bejo ${ }^{\mathrm{b}, \mathrm{c}}$, Raha \\ Abdul Rahimª, Nurulfiza Mat Isa ${ }^{a, c^{*}}$ \\ ${ }^{a}$ Department of Cell and Molecular Biology, Faculty of Biotechnology and Biomolecular Sciences, Universiti Putra Malaysia, Malaysia \\ ${ }^{b}$ Department of Veterinary Pathology and Microbiology, Faculty of Veterinary Medicine, Universiti Putra Malaysia, Malaysia \\ 'Laboratory of Vaccines and Immunotherapeutics, Institute of Bioscience, Universiti Putra Malaysia, 43400 Serdang, Selangor Darul Ehsan, \\ Malaysia \\ ${ }^{d}$ Laboratory of UPM-MAKNA Cancer Research, Institute of Bioscience, Universiti Putra Malaysia, 43400 Serdang, Selangor Darul Ehsan, \\ Malaysia
}

Received 1st October 2018 / Accepted 11th September 2019

\begin{abstract}
Chitosan is a biodegradable, non-toxic polysaccharide that is extensively studied as a biocompatible vector for gene and drug delivery. However, the fabrication of chitosan nanoparticle (CNP) is usually encountered with a wide size distribution and poor particle stability, which unfortunately limits their role for certain biological applications. This study reports the synthesis and characterisation of CNPs under optimised conditions. The CNPs were synthesised via ionic gelation process utilizing tripolyphosphate (TPP) as a cross-linking agent. The particle size and morphology of samples were subsequently evaluated using dynamic light scattering (DLS), electron microscopy and Fourier-transform infrared spectroscopy (FTIR). Findings arising from this study showed the optimised nanoparticles exhibited spherical shaped CNPs with a size range from 4 to $25 \mathrm{~nm}$ which lays the foundation for further applications.
\end{abstract}

Keywords: Chitosan nanoparticles, dynamic light scattering, Fourier-transform infrared spectroscopy, transmission electron microscope

\section{INTRODUCTION}

The delivery of genes and nucleic acid, especially non-viral routes have been developed as a powerful and popular approach for gene therapy and DNA vaccination in the treatment as well as control of diseases that are likely to impact clinical medicine and biotechnology (Luo and Saltzman, 2000). The ability to transfer foreign DNA into cells safely and efficiently is a fundamental goal for such applications. Therefore, there lies a growing need to improve its current effectiveness in terms of efficient delivery and sustained antigen release. In order to achieve this, research has greatly skewed towards the use of biodegradable polymers as nanoparticle-based carriers to deliver and increase intracellular accumulation of nucleic acids.

Such a polymer includes chitin, a natural nitrogenous polysaccharide conferring white and hard structures that make up the exoskeleton of insects, crustaceans and some fungi (Kumar, 2000). Deacetylation of chitin produces a derivative known as chitosan that has been widely

*Author for correspondence: Dr. Nurulfiza Mat Isa, Department of Cell and Molecular Biology, Faculty of Biotechnology and Biomolecular Sciences, Universiti Putra Malaysia, 43400 UPM Serdang, Selangor Darul Ehsan, Malaysia. Email-nurulfiza@upm.edu.my 
used for many applications (Paul, 2000; Muzzarelli \& Muzzarelli, 2005; Dash et al., 2011). Chitosan is a semi synthetic amino polysaccharides of glucosamine and $\mathrm{N}$-acetyl-d-glucosamine linked together by $\beta(1,4)$ glycosidic bond (Gan \& Wang, $2007)$ that possess favourable biological characteristics such as biodegradability, biocompatibility, low toxicity, haemostatic, bacteriostatic, fungistatic, anti-carcinogenic and anti- cholesteremic properties. Chitosan has been developed as a drug delivery carrier by complexing chitosan nanoparticles (CNPs) for the delivery of particular drugs for cancer treatments (Bugnicourt \& Ladavière, 2016). CNPs also exhibit great performance as a non-viral vector for gene delivery (Fan et al., 2012).

Various methods have been employed to synthesise CNPs such as emulsion cross linking (Akbuga \& Durmaz, 1994), coacervation or precipitation (Mao et al., 2001), emulsion droplet coalescence (Tokomitsu et al., 1999), and ionic gelation (Fan et al., 2012). Ionic gelation was found to be the most favourable due to its safer and more robust route of synthesis (Fan et al., 2012). The process involves physical cross linking instead of chemical cross linking, which are often toxic to organisms (Fan et al., 2012). A spherical particle known as nanoparticles was produced after both oppositely charged species of chitosan and TPP forming a complex (Dash et al., 2011). This study was aimed to synthesise and characterise CNPs.

\section{MATERIALS AND METHODS}

\section{Synthesis of chitosan/TPP nanoparticles}

Chitosan nanoparticles were prepared by ionic gelation routes, with slight modifications based on methods described by Calvo et al. (1997). To obtain the smallest nanoparticle size of chitosan, experimental optimisation was performed by changing the concentration of chitosan and TPP solutions at a 1:1 ratio $(\mathrm{v} / \mathrm{v})$ with different volumes of TPP solutions.

The optimisation procedures were performed by first dissolving the chitosan powder in $0.5 \%$ $(\mathrm{v} / \mathrm{v})$ acetic acid solution to a concentration of $0.1,0.5$ and $1.0 \mathrm{mg} / \mathrm{mL}$ at $\mathrm{pH} 5.0$. Subsequently, the TPP solution was prepared by dissolving TPP powder to concentrations ranging from $0.1,0.5$ and $1.0 \mathrm{mg} / \mathrm{mL}$ in ultrapure water at $\mathrm{pH} 7.4$. Both solutions were then stirred overnight at room temperature. The chitosan solution was then passed through a $0.45 \mu \mathrm{m}$ (Millipore, USA) syringe filter. To produce the nanoparticle suspension, synthesis was done using a series of chitosan and TPP concentrations $(0.1,0.5$ and 1.0 $\mathrm{mg} / \mathrm{mL}$ ) at a $1: 1$ concentration ratio by dropwise additions of TPP at different volumes (25, 50, 100, 200, 250 and $300 \mu \mathrm{L}$ ) into $2 \mathrm{~mL}$ chitosan solution at the rate of 20-40 drops per minutes (Muhammadpourdounighi et al., 2010). The suspensions were then subjected to further characterisation.

\section{Physicochemical characterisation of CNPs and $p N Z$ : VP2IL15-CNPs}

The particle size distribution of all nanoparticle samples was measured using dynamic light scattering (DLS) on a High-Performance Particle Sizer 3000 (Malvern Instrument, UK). Approximately $100 \mu \mathrm{L}$ of sample firstly was resuspended in $900 \mu \mathrm{L}$ ultra-pure water before analysis. Particle size mean values were obtained from the triplicate analysis of three different batches. Morphological and particle sizes of nanoparticle samples were cross-analysed by electron microscopy using H-7100 TEM (Hitachi, Japan). Nanoparticles were dropped on a parafilm and a carbon coated grid (Agar Scientific, UK) was placed on the CNPs drop, left for 5 minutes before fixing in $2 \%$ phosphotungstic acid (PTA) (Sigma, USA) for 5 minutes. Excess liquid was then removed from the grid using a Whatman paper (GE Healthcare, UK) and dried in a desiccator before viewing. The infrared spectra of CNPs and pNZ: VP2IL15-CNPs were then recorded on a Perkin-Elmer FTIR spectrometer (SPECTRUM 1000).

\section{RESULTS}

\section{Synthesis of CNPs}

As an optimisation, three different concentrations of chitosan and TPP $(0.1,0.5$ and $1.0 \mathrm{mg} / \mathrm{mL})$ were prepared and both solutions were combined using 1:1 concentration ratio with a series of TPP volumes $(25,50,100,200,250$ and $300 \mu \mathrm{L})$ into 2 
$\mathrm{mL}$ chitosan and z-average particle size were measured by DLS. The result (Figure 1) showed all the three different combinations of chitosan and TPP solutions resulted to chitosan with nanoparticle sizes ranging from $45 \mathrm{~nm}$ to less than $300 \mathrm{~nm}$. Combination of chitosan and TPP at 1.0 $\mathrm{mg} / \mathrm{mL}$ showed the nanoparticle sizes.

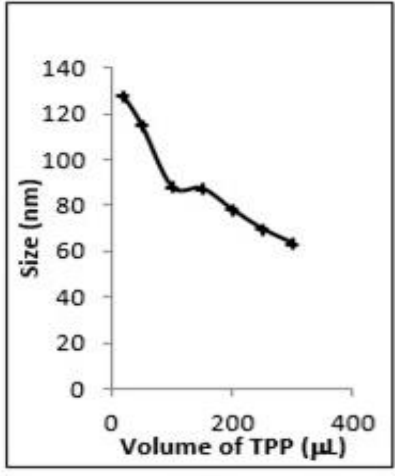

(a)

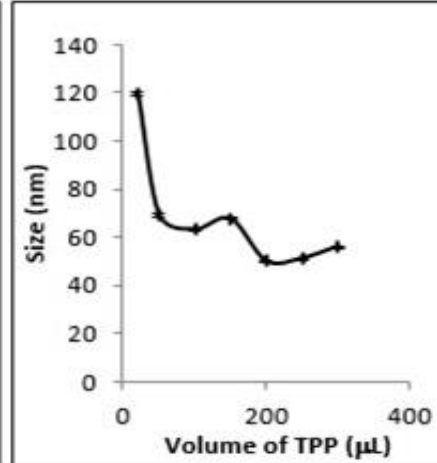

(b)

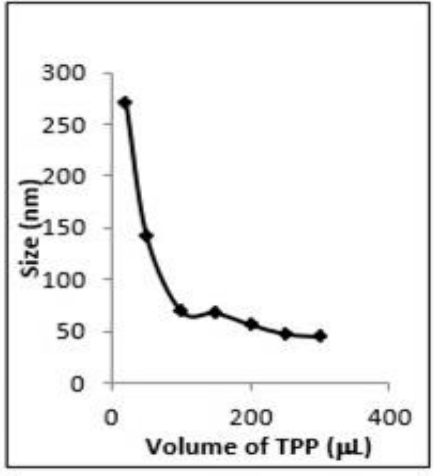

(c)

Figure 1. (a) Size of CNPs generated with combination of chitosan and TPP solutions at concentration of $0.1 \mathrm{mg} / \mathrm{mL}$; (b) Size of CNPs generated with combinations of chitosan and TPP solutions at concentration of $0.5 \mathrm{mg} / \mathrm{mL}$; (c) Size of CNPs generated with combinations of chitosan and TPP solutions at concentration of $1.0 \mathrm{mg} / \mathrm{mL}$.

\section{Characterisation of CNPs by TEM}

The morphology of optimised CNPs was observed by TEM. Typical CNPs showed spherical and polydisperse nature as shown in Figure 2. The CNPs conferred a round shape and smooth surfaces with sizes ranging from $3.22 \mathrm{~nm}$ to $23.69 \mathrm{~nm}$.

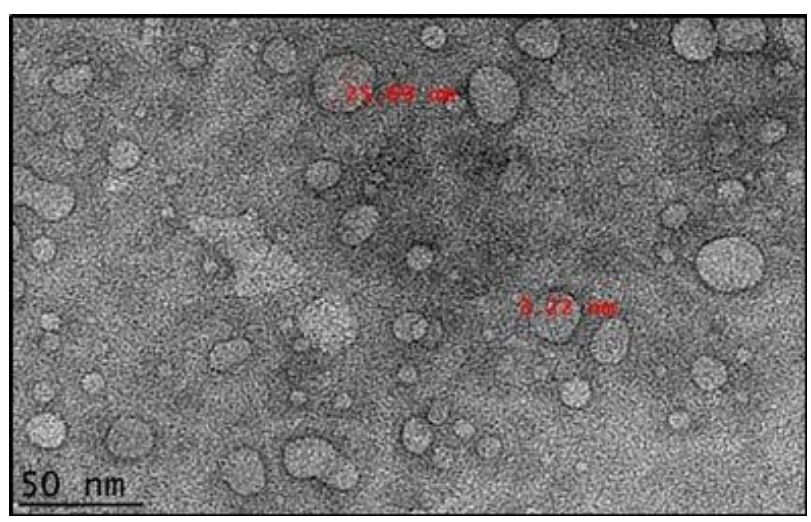

Figure 2. Transmission electron microscopy micrograph of CNPs.

\section{Characterisation of CNPs by TEM}

The result of FTIR spectra of CNPs was shown in Figure 3. CNPs spectrum showed a symmetric stretch of C-O-C that was observed around 1086 $\mathrm{cm}^{-1}$. Peak with wavelength 893 and $648 \mathrm{~cm}^{-1}$ signifies the presence of R-CH group. A complex formation of chitosan and TPP polyanions was identified with the presence of peaks at $1560 \mathrm{~cm}^{-1}$ indicated the presence of amino group that found in CNPs.

\section{DISCUSSION}

Chitosan nanoparticles have been extensively studied as a drug delivery carrier for various antibiotics, anti-hypersensitive agents, anticancer agents, proteins, peptide drugs, and vaccines (Kunjachan et al., 2014). Other types of biomolecules have been investigated to be developed as nanocarrier, such as micelles, polymeric nanoparticles, polymer-drug conjugates and liposomes. Results from optimised CNPs conditions indicated that increased chitosan and TPP concentrations resulted in a subsequent increase towards the size of the nanoparticles produced; indicating that formation of CNPs at different sizes were somewhat dependent on specific concentrations of chitosan and TPP. CNPs measured by both DLS ( $50 \mathrm{~nm})$ and TEM (3-23 nm). Different sizes of CNPs obtained between TEM and DLS was observed. The differences between DLS and TEM occur due to swelling of the CNPs in liquid media and DLS gives a larger nanoparticle size while TEM gives an actual diameter of nanoparticles in dry state. The part of aggregation of the chitosan/TPP nanoparticles is probably because that the 
hydrogen bonding interactions between chitosan nanoparticles gradually become dominant in the drying process of $5 \mathrm{~nm}$ could in fact make a huge difference on the properties and biological functions. This result corresponded with findings by Cavlo et al. (1997) showed that the CNPs were synthesised at specific concentration of chitosan and TPP. In this study, optimum size of CNPs was achieved at $1.0 \mathrm{mg} / \mathrm{mL}$ of chitosan and TPP.

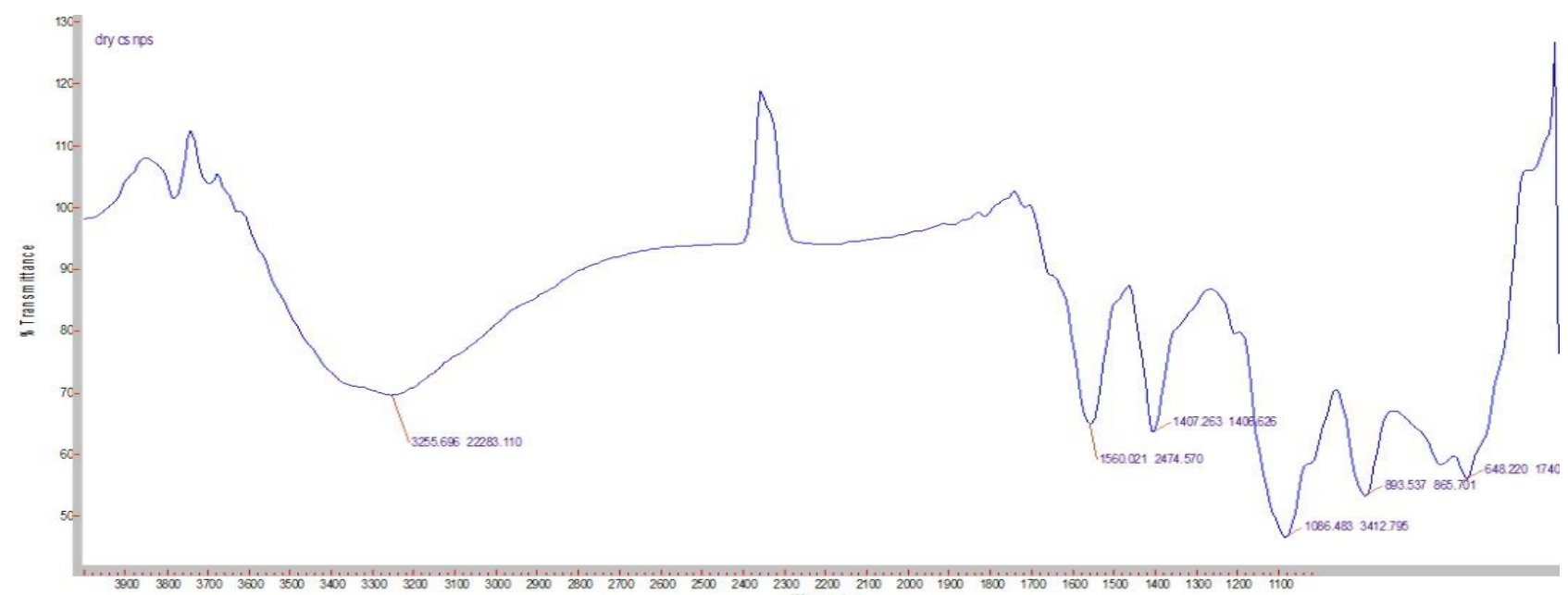

Figure 3. Fourier transform infrared spectra (FTIR) of CNPs.

A gradual decrease of nanoparticle size was obtained with increasing volumes of TPP added to the chitosan solution. Studies by Fan et al. (2012) suggested a similar finding, where nanoparticle size decreased with increasing volumes of TPP indicating that protonated amino group of chitosan was neutralized by TPP anions. The best performed combination of chitosan and TPP was at $1.0 \mathrm{mg} / \mathrm{mL}$ with addition of $300 \mu \mathrm{L}$ of TPP. The size of CNPs was found to be less than $50 \mathrm{~nm}$ as elucidated by DLS and TEM analyses. Nanoparticle sizes obtained in this study corresponded well with previous study (Fan et al., 2012) which exhibited spherical shape with particle size ranges from 30-50 $\mathrm{nm}$.

Similar result also obtained in previous studies performed by ionic gelation method (Vimal et al., 2012; Gan \& Wang, 2007; Xu \& Du, 2003). FTIR analysis of CNPs showed a peak at $1560 \mathrm{~cm}^{-1}$, indicating the N-H bending vibration that form as a result of chitosan and TPP interaction. Such a peak occurrence was also reported in another study by Muhammadpourdounighi et al. (2010).

\section{CONCLUSION}

Prabha et al. (2002) predicted smaller nanoparticles are more efficient in delivering drugs into tissues or cells compared to larger nanoparticle sizes. In this study, we successfully synthesised small size of CNPs which could be a potential candidate for further applications.

\section{ACKNOWLEDGEMENTS}

This work was supported by the ScienceFund research grant number 02-01-04-SF2033 from the Ministry of Science, Technology and Innovation of Malaysia (MOSTI) and Research University Grant Scheme, vote number of 9451300, Universiti Putra Malaysia, Malaysia. The first author was financially supported throughout her study by MyBrain15 MyMaster.

\section{REFERENCES}

Akbuga, J., \& Durmaz, G. 1994. Preparation and evaluation of cross-linked chitosan microspheres containing furosemide. International Journal of Pharmaceutics 111(3): 217 222.

Bugnicourt, L., \& Ladavière, C. 2016. Interests of chitosan nanoparticles ionically cross-linked with tripolyphosphate for biomedical applications. Progress in Polymer Science 60: 1 17.

Calvo, P., Remunan-Lopez, C., Vila-Jato, J. L., \& Alonso, M. J. 1997. Novel hydrophilic chitosan-polyethylene oxide 
nanoparticles as protein carriers. Journal of Applied Polymer Science 63(1): 125-132.

Dash, M., Chiellini, F., Ottenbrite, R. M., \& Chiellini, E. 2011. Chitosan-A versatile semi-synthetic polymer in biomedical applications. Progress in Polymer Science 36(8), 9811014.

Fan, W., Yan, W., Xu, Z., \& Ni, H. 2012. Formation mechanism of monodisperse, low molecular weight chitosan nanoparticles by ionic gelation technique. Colloids and Surfaces B: Biointerfaces 90: 21-27.

Gan, Q., \& Wang, T. 2007. Chitosan nanoparticle as protein delivery carrier-systematic examination of fabrication conditions for efficient loading and release. Colloids and Surfaces B: Biointerfaces 59(1): 24-34.

Kumar, M. N. R. 2000. A review of chitin and chitosan applications. Reactive and functional polymers 46(1): 1-27.

Kunjachan, S., Jose, S., \&Lammers, T. 2014. Understanding the mechanism of ionic gelation for synthesis of chitosan nanoparticles using qualitative techniques. Asian Journal of Pharmaceutics (AJP) 4:(2).

Luo, D., \& Saltzman, W. M. 2000. Synthetic DNA delivery systems. Nature Biotechnology 18(1): 33-37.

Mao, H. Q., Roy, K., Troung-Le, V. L., Janes, K. A., Lin, K. Y., Wang, Y., \& Leong, K. W. 2001. Chitosan-DNA nanoparticles as gene carriers: synthesis, characterization and transfection efficiency. Journal of Controlled Release 70(3): $399-421$.

Mohammadpourdounighi, N., Behfar, A., Ezabadi, A., Zolfagharian, H., \& Heydari, M. 2010. Preparation of chitosan nanoparticles containing Najanajaoxiana snake venom. Nanomedicine: Nanotechnology, Biology and Medicine 6(1): 137-143.

Muzzarelli, R. A. A., \& Muzzarelli, C. 2005. Chitosan chemistry: relevance to the biomedical sciences. In Polysaccharides I pp: 151-209. Springer Berlin Heidelberg.

Paul, W., \& Sharma, C. P. 2000. Chitosan, a drug carrier for the 21 st century: a review. STP Pharma Sciences 10(1): 5-22.

Prabha, S., Zhou, W. Z., Panyam, J., \& Labhasetwar, V. 2002. Sizedependency of nanoparticle-mediated gene transfection: studies with fractionated nanoparticles. International Journal of Pharmacentics 244(1): 105-115.

Tokumitsu, H., Ichikawa, H., \& Fukumori, Y. 1999. Chitosangadopentetic acid complex nanoparticles for gadolinium neutron-capture therapy of cancer: preparation by novel emulsion-droplet coalescence technique and characterization. Pharmaceutical Research 16(12): 1830-1835.

Vimal, S., Majeed, S. A., Taju, G., Nambi, K. S. N., Raj, N. S., Madan, N., Farook, M. A., Rajkumar, T., Gopinath, D., \&Hameed, A. S. 2013. Chitosan tripolyphosphate (CS/TPP) nanoparticles: Preparation, characterization and application for gene delivery in shrimp. ActaTropica 128(3): 486-493.

Xu, Y., \& Du, Y. (2003). Effect of molecular structure of chitosan on protein delivery properties of chitosan nanoparticles. International Journal of Pharmaceutics 250(1): 215-226. 